\title{
Exclusive open-charm near-threshold cross sections in a coupled-channel approach
}

\author{
Timofey $\mathrm{Uglov}^{1, *}$ \\ ${ }^{1}$ P. N. Lebedev Physical Institute of the Russian Academy of Sciences
}

\begin{abstract}
Data on 7 open-charm channels collected by the Belle Collaboration are analyzed simultaneously using a unitary approach based on a coupledchannel model in a wide energy range $\sqrt{s}=3.7-4.7 \mathrm{GeV}$. The resulting fit provides a remarkably good overall description of the line shapes in all studied channels. Parameters of 5 vector charmonium resonances are extracted from the fit. It is demonstrated, that this approach could be used account for all exclusive channels and thus solve the long-term problem of the charmonium spectra near threshold.
\end{abstract}

\section{Introduction}

Four vector charmonia above the open-charm threshold, $\psi(3770), \psi(4040), \psi(4160), \psi(4415)$, were discovered forty years ago in the $e^{+} e^{-}$annihilation as peaks in the total hadronic cross section [1-5]. Only thirty years later parameters of these states were updated from a naive combined fit [6] for the Crystal Ball [7] and the BES data [8]. In 2008 BES fitted the total hadronic cross section (in terms of the ratio $R$ ) [8] taking into account the interference and the relative phases between the exclusive decays of the $\psi$-resonances [9]. As BES used model predictions for the $\psi$ 's decays into the two-body charmed final states, the obtained parameters remain model-dependent.

A comprehensive study of the exclusive $e^{+} e^{-}$cross sections to various open-charm final states could help one to extract parameters of the $\psi$ states in a model-independent way and therefore to shed light on the nature of the $Y$ family. Such cross sections were first measured by Belle [13, 15-19] and BaBar [20-22] at the $B$-factories, using the ISR in a wide energy region $\sqrt{s}=3.7 \div 5.0 \mathrm{GeV}$, and by CLEO [23], using the energy scan over $\sqrt{s}=3.97 \div$ $4.26 \mathrm{GeV}$ at the charm factory. BaBar performed fits to the measured two-body $D \bar{D}, D \bar{D}^{*}$, $D^{*} \bar{D}^{*}$, and $D_{s}^{(*)+} D_{s}^{(*)-}$ cross sections with the parameters of the $\psi$ states fixed to the Particle Data Group (PDG) [24] values. From this study only the ratios of the branching fractions for the $\psi(4040), \psi(4160)$, and $\psi(4415)$ decays to the $D \bar{D}, D \bar{D}^{*}$, and $D^{*} \bar{D}^{*}$ were extracted. CLEO compared the $e^{+} e^{-}$cross sections to the same open-charm meson pairs with the predictions of an updated potential model [14] that appeared to fail to describe the data. Belle presented not only the two-body but also the three-body $D^{0} D^{(*)-} \pi^{+}$and the charmed baryon $\Lambda_{c}^{+} \Lambda_{c}^{-}$cross sections and demonstrated that the sum of the measured partial cross sections almost saturates the total hadronic cross section.

Many attempts were made to describe the measured exclusive cross sections [25-34].

\footnotetext{
*e-mail: uglov.timofey@gmail.com
} 
Below a coupled-channel fit to the exclusive cross sections of the $e^{+} e^{-}$annihilation to various open-charm final states is presented. The detailed explanation of the fit technique is given in [35]. The most accurate and coherent measurements in the full $\sqrt{s}$ interval below $5 \mathrm{GeV}$ for numerous two-body final states in the $e^{+} e^{-}$annihilation was presented by the Belle Collaboration. For the further analysis the Belle data, available at the Durham database [36], are used.

As was demonstrated by Belle, the sum of the four channels $\left(D \bar{D}, D \bar{D}^{*}, D^{*} \bar{D}^{*}, D \bar{D} \pi\right)$ well saturates the inclusive hadronic cross section in the region $\sqrt{s} \lesssim 4.7 \mathrm{GeV}$, so we confine ourselves by considering these four channels and this region only. Since the charged and the neutral modes in each channel are related to each other by the isospin symmetry, we only distinguish between the $D^{+} D^{-}$and $D^{0} \bar{D}^{0}$ modes explicitly since they are presented separately by Belle.

For the $D \bar{D} \pi$ final state, Belle presented the cross section for the $D^{0} D^{-} \pi^{+}$mode and demonstrated that this final state is dominated by the contribution from the two-body mode $D \bar{D}_{2}$. To convert the measured $D^{0} D^{-} \pi^{+}$cross section into the $D \bar{D}_{2}$ one we correct the former by the ratio of the branching fractions $\mathcal{B}\left(D_{2} \rightarrow D \pi\right) /\left(\mathcal{B}\left(D_{2} \rightarrow D \pi\right)+\mathcal{B}\left(D_{2} \rightarrow D^{*} \pi\right)\right)$ [24].

Finally, when identifying the open-charm channels it should be taken into account that 3 different final states are allowed for the $D^{*} \bar{D}^{*}$ channels, namely, the $P$ wave with $S=0$, the $P$ wave with $S=2$, and the $F$ wave with $S=2$. We therefore stick to the set of 16 channels, thus counting the charge- and isospin-conjugated final states as independent ones,

$$
\begin{array}{cccccc}
D \bar{D} & D \bar{D}^{*} & D_{2} \bar{D} & {\left[D^{*} \bar{D}^{*}\right]_{S=0}^{P}} & {\left[D^{*} \bar{D}^{*}\right]_{S=2}^{P}} & {\left[D^{*} \bar{D}^{*}\right]_{S=2}^{F}} \\
2 \text { channels } & 4 \text { channels } & 4 \text { channels } & 2 \text { channels } & 2 \text { channels } & 2 \text { channels }
\end{array}
$$

The channels are labelled by latin letters $i, j$, and so on. All parameters (but the charged and neutral meson masses!) in the isospin-cojugated channels are taken equal to each other.

Reactions $e^{+} e^{-} \rightarrow D^{(*)} \bar{D}^{(*)}$ studied in this work are expected to proceed through the 5 intermediate vector resonances,

$$
\psi(2 S), \psi(3770), \psi(4040), \psi(4160), \psi(4415),
$$

which we denote as $\psi$ 's and label by greek letters $\alpha, \beta$, and so on.

The aim of the present study is to establish a proper general formalism and to determine the parameters of the vector resonances $\psi$ enumerated in (2) from a simulations fit for all exclusive open-charm channels measured by Belle - see (1). The cornerstone of the approach used is unitarity preserved at every stage of the data analysis.

\section{Coupled-channel model}

The traditional way to analyse the experimental data is to use individual Breit-Wigner distributions for each peak combined with a suitable background. It has to be noticed however that such an approach can provide only very limited information on the states under study. Instead in our analysis we are taking rid of $K$-matrix formalism, which conservs unitarity.

The amplitude reads

$$
A_{i j}=\sum_{\alpha \beta} G_{i \alpha}(s) P_{\alpha \beta}(s) G_{j \beta}(s)
$$

with

$$
\left(P^{-1}(s)\right)_{\alpha \beta}=\left(M_{\alpha}^{2}-s\right) \delta_{\alpha \beta}-i \sum_{m} G_{m \alpha} G_{m \beta}, \quad G_{i \alpha}^{2}(s)=g_{i \alpha}^{2} \frac{k_{i}^{2 l_{i}+1}}{\sqrt{s}} \theta\left(s-s_{i}\right)
$$


where $i$ and $j$ run over hadronic channels and $\alpha$ labels the bare $\bar{c} c$ states with the masses $M_{\alpha}$.

The couplings $g_{i \alpha}$ are defined as follows. The width $\Gamma_{i \alpha}$ for the vector $\psi_{\alpha}$ decaying into the $i$-th open-charm channel $\left[D^{(*)} \bar{D}^{(*)}\right]_{i}$ is given by

$$
\Gamma_{i \alpha} \equiv \Gamma\left(\psi_{\alpha} \rightarrow\left[D^{(*)} \bar{D}^{(*)}\right]_{i}\right)=\frac{g_{i \alpha}^{2}}{M_{\alpha}^{2}}\left[p_{i}\left(M_{\alpha}\right)\right]^{2 l_{i}+1},
$$

where $p_{i}\left(M_{\alpha}\right)=\lambda^{1 / 2}\left(M_{\alpha}^{2}, m_{D_{i}^{(*)}}^{2}, m_{\bar{D}_{i}^{(*)}}^{2}\right) /\left(2 M_{\alpha}\right)$ is the centre-of-mass momentum and $l_{i}$ is the angular momentum in the final state. In particular, $l_{i}=1$ for $i=D^{+} D^{-}, D^{0} \bar{D}^{0}, D^{+} D^{*-}$, $\left[D^{*+} D^{*-}\right]_{S=0}^{P}$, and $\left[D^{*+} D^{*-}\right]_{S=2}^{P}$, then $l_{i}=2$ for the $D_{2}^{+} D^{-}$final state, and, finally, $l_{i}=3$ in the $\left[D^{*+} D^{*-}\right]_{S=2}^{F}$ channel.

In the Vector Dominance Model (VDM) the $\psi_{\alpha}$ 's electronic width reads

$$
\Gamma_{e \alpha} \equiv \Gamma\left(\psi_{\alpha} \rightarrow e^{+} e^{-}\right)=\frac{\alpha g_{e \alpha}^{2}}{3 M_{\alpha}^{3}} .
$$

Therefore the total cross section for the annihilation process $e^{+} e^{-} \rightarrow\left[D^{(*)} \bar{D}^{(*)}\right]_{i}$,

$$
\sigma_{i}(s)=\frac{4 \pi \alpha}{s^{5 / 2}}\left[p_{i}(s)\right]^{2 l_{i}+1}\left|\sum_{\alpha, \beta} g_{e \alpha} P_{\alpha \beta}(s) g_{i \beta}\right|^{2},
$$

where the inverse propagators matrix $P^{-1}$ is defined in (4).

The set of the 16 open-charm channels (1) can be described with 40 parameters,

$$
\left\{M_{\alpha}, \Gamma_{e \alpha}, g_{i \alpha}\right\}, \quad \alpha=\overline{1,5}, \quad i=\overline{1,16},
$$

where, for convenience, we use the electronic widths $\Gamma_{e \alpha}$ as the free parameters instead of the couplings $g_{e \alpha}$ - see their interrelation in (6).

To diminish the influence of indistinguishability between 3 possible $D^{(*)} \bar{D}^{(*)}$ helicity components we employ some heavy-quark spin symmetry (HQSS) constraints for the couplings. We assume in what follows $\psi(2 S) \equiv \psi_{1}, \psi(4040) \equiv \psi_{3}$, and $\psi(4415) \equiv \psi_{5}$ to be predominantly ${ }^{3} S_{1}$ states and $\psi(3770) \equiv \psi_{2}$ and $\psi(4160) \equiv \psi_{4}$ to be predominantly ${ }^{3} D_{1}$ states, and relate the $P$-wave couplings in the $D^{*} \bar{D}^{*}$ channels,

$$
\begin{aligned}
& g_{\left[D^{*} \bar{D}^{*}\right]_{P 2}, \alpha}=-\sqrt{20} g_{\left[D^{*} \bar{D}^{*}\right]_{P 0}, \alpha}, \quad \alpha=1,3,5, \\
& g_{\left[D^{*} \bar{D}^{*}\right]_{P 0}, \alpha}=-\sqrt{5} g_{\left[D^{*} \bar{D}^{*}\right]_{P 2}, \alpha}, \quad \alpha=2,4,
\end{aligned}
$$

so that the number of the remaining free parameters of the model is reduced to 35 . For the masses of the $D$ mesons involved we use the standard PDG values [24].

\section{Fit for the experimental data}

We have checked the consistency of the model with the experimental data and performed a simultaneous fit for the open-charm exclusive cross sections in the interval corresponding to the known $\psi$ states. The fit minimises the function $\chi_{\exp }^{2}$ defined as a sum of $\left(\sigma_{\exp }-F F\right)^{2} / \delta_{\exp }^{2}$ over all Belle experimental points. Here $F F$ is the Fitting Function for the given channel - see (7) - while $\sigma_{\exp }$ and $\delta_{\text {exp }}$ are the experimental value and the corresponding error, respectively. The statistical and systematic experimental errors of the Belle data are summed in squared.

We take into account contributions of all $5 \psi$-resonances from (2) and treat the coupling constants of all 5 states to all channels as the free parameters of the fit (with the constrains 

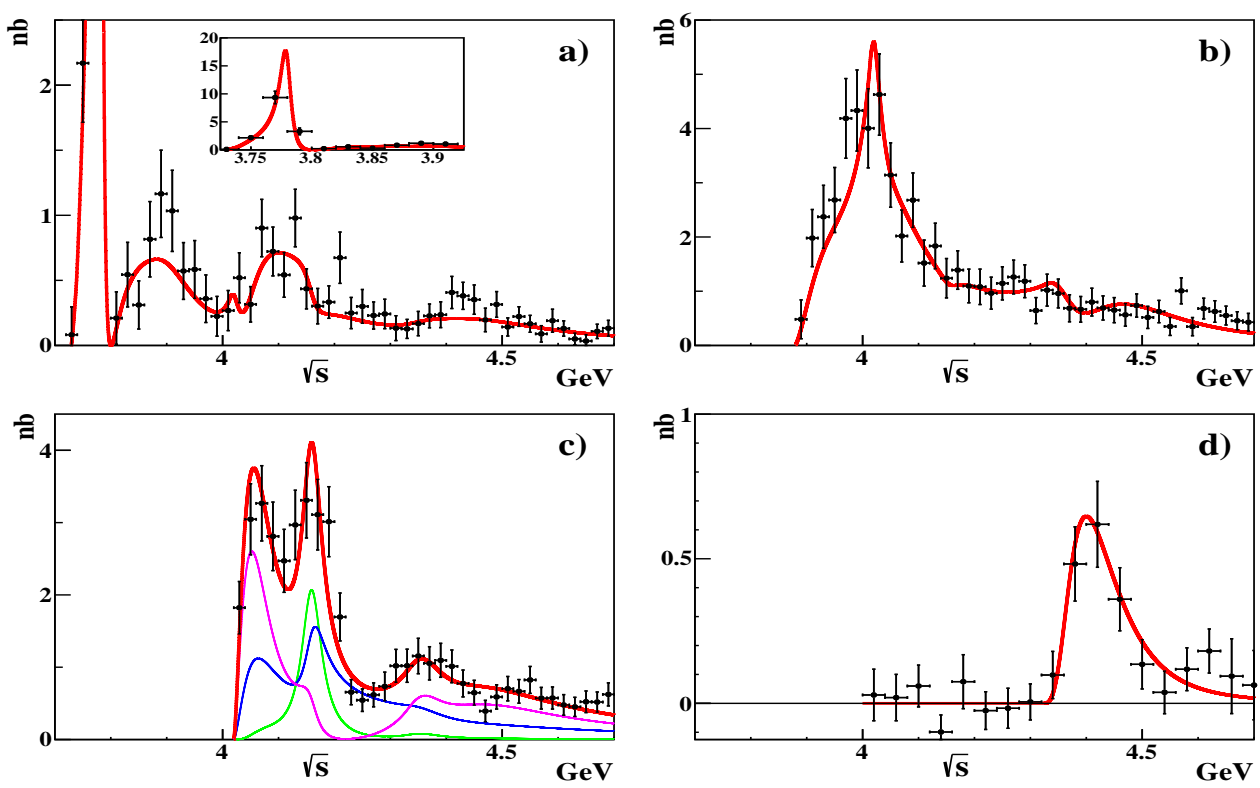

Figure 1. Exclusive cross sections for the $e^{+} e^{-} \rightarrow D \bar{D}\left(\left[e^{+} e^{-} \rightarrow D^{+} D^{-}\right]+\left[e^{+} e^{-} \rightarrow D^{0} \bar{D}^{0}\right]\right)$ (plot (a)), $e^{+} e^{-} \rightarrow D^{+} D^{*-}$ (plot (b)), $e^{+} e^{-} \rightarrow D^{*+} D^{*-}$ (plot (c)), and $e^{+} e^{-} \rightarrow D \bar{D} \pi\left(\left[e^{+} e^{-} \rightarrow D^{0} D^{-} \pi^{+}\right]+\left[e^{+} e^{-} \rightarrow\right.\right.$ $\left.\bar{D}^{0} D^{+} \pi^{-}\right]$) (plot (d)). In all plots, the points with the error bars represent the Belle data [36] and the red curves show the fit results. In plot (c), the blue, the green, and the magenta thin curves represent the $P$-wave with $S=0$, the $P$-wave with $S=2$, and the $F$-wave with $S=0$ contributions in $D^{*+} D^{*-}$ final state, respectively.

from the HQSS imposed). The coupling constants to the neutral and the charged modes are set equal to each other. We also assume that the $D \bar{D} \pi$ final state is dominated by the $D \bar{D}_{2}$ intermediate state.

We fix $\psi(2 S)$ mass, its electronic and total width to the PDG values [24], so that the total number of the free parameters in the fit is eventually reduced to 33 .

To avoid the fit to blow up the resonances and find unphysical minima we add the extra term to the $\chi^{2}$ :

$$
\chi_{\text {tot }}^{2}=\chi_{\exp }^{2}+\sum_{\alpha=1}^{5}\left\{\left(\frac{M_{\alpha}-M_{\alpha}^{\mathrm{PDG}}}{50 \mathrm{MeV}}\right)^{2}+\left(\frac{\Gamma_{e \alpha}-\Gamma_{e \alpha}^{\mathrm{PDG}}}{0.5 \mathrm{MeV}}\right)^{2}+\left(\frac{\sum_{i=1}^{16} \Gamma_{i \alpha}}{200 \mathrm{MeV}}\right)^{2}\right\}
$$

where the indices $i$ and $\alpha$ run over the open-charm channels and the $\psi$-resonances, as given in (1) and (2), respectively. The formulae for the widths are given in (5) and (6).

Finally, we fit 191 experimental points in all 5 open-charm channels with the fitting function which contains 33 free parameters. It turns out that $\chi_{\text {tot }}^{2}$ possesses multiple local minima separated by barriers in the parameter space.

The best fit found has $\chi_{\exp }^{2}=158$, that is it is almost perfect. The line shapes which correspond to this best fit are plotted in Figs. 1 and the set of parameters is listed in Table 1. For convenience, we also quote the partial widths of the $\psi$ 's in all studied channels - see (5). 
Table 1. Parameters of the $\psi$-resonances extracted from the fit for the Belle data. Parameters marked with the asterisk are artificially fixed to the PDG values.

\begin{tabular}{|c|c|c|c|c|c|}
\hline & $\psi_{1}$ & $\psi_{2}$ & $\psi_{3}$ & $\psi_{4}$ & $\psi_{5}$ \\
\hline PDG name & $\psi(2 S)$ & $\psi(3770)$ & $\psi(4040)$ & $\psi(4160)$ & $\psi(4415)$ \\
\hline$M, \mathrm{MeV}$ & $3686^{*}$ & $3782 \pm 1$ & $4115 \pm 14$ & $4170 \pm 7$ & $4515 \pm 18$ \\
\hline \multicolumn{6}{|c|}{ Coupling constants $g_{i \alpha}\left(\alpha=1 \ldots 5, i=D \bar{D}, D \bar{D}^{*}\right.$, etc - see $\left.(1)\right)$} \\
\hline$D \bar{D}$ & $3.0 \pm 0.3$ & $-1.8 \pm 0.3$ & $-0.1 \pm 0.1$ & $0.3 \pm 0.1$ & $-0.1 \pm 0.1$ \\
\hline$D \bar{D}^{*}$ & $-4.7 \pm 0.5$ & $-3.1 \pm 0.3$ & $2.4 \pm 0.2$ & $-0.0 \pm 0.7$ & $-0.7 \pm 0.2$ \\
\hline$\left[D^{*} \bar{D}^{*}\right]_{S=0}^{P}$ & $4.8 \pm 0.5$ & $6.9 \pm 0.9$ & $-0.1 \pm 0.2$ & $0.6 \pm 0.5$ & $-0.3 \pm 0.1$ \\
\hline$\left[D^{*} \bar{D}^{*}\right]_{S=2}^{P}$ & $-21.7 \pm-2.3$ & $-3.1 \pm-0.4$ & $0.5 \pm 0.9$ & $-0.3 \pm-0.2$ & $1.5 \pm-0.3$ \\
\hline$\left[D^{*} \bar{D}^{*}\right]_{S=2}^{F}, \mathrm{MeV}^{-2}$ & $62.2 \pm 15.1$ & $-1.6 \pm 5.4$ & $-1.0 \pm 2.8$ & $8.0 \pm 1.4$ & $0.2 \pm 0.6$ \\
\hline$D_{2} \bar{D}, \mathrm{MeV}^{-1}$ & $-8.2 \pm 29.3$ & $25.2 \pm 7.7$ & $-23.5 \pm 3.3$ & $-1.0 \pm 7.4$ & $-1.5 \pm 1.4$ \\
\hline \multicolumn{5}{|c|}{ Partial decay widths $\Gamma_{i \alpha}, \mathrm{MeV}$} & \\
\hline$e^{+} e^{-}$ & $2.354^{*}$ & $0.2 \pm 0.0$ & $1.6 \pm 0.3$ & $0.7 \pm 0.4$ & $1.4 \pm 0.3$ \\
\hline$D^{+} D^{-}$ & - & $5.6 \pm 1.7$ & $0.4 \pm 0.8$ & $4.3 \pm 2.6$ & $0.5 \pm 1.0$ \\
\hline$D^{0} \bar{D}^{0}$ & - & $7.5 \pm 2.2$ & $0.4 \pm 0.8$ & $4.5 \pm 2.7$ & $0.5 \pm 1.0$ \\
\hline$D^{+} D^{*-}$ & - & - & $110.7 \pm 23.5$ & $0.0 \pm 0.5$ & $32.8 \pm 17.4$ \\
\hline$\left[D^{*} \bar{D}^{*}\right]_{S=0}^{P}$ & - & - & $0.1 \pm 0.2$ & $3.6 \pm 6.5$ & $5.9 \pm 2.6$ \\
\hline$\left[D^{*} \bar{D}^{*}\right]_{S=2}^{P}$ & - & - & $1.2 \pm 6.8$ & $0.7 \pm 0.3$ & $118.0 \pm 729.4$ \\
\hline$\left[D^{*} \bar{D}^{*}\right]_{S=2}^{F}$ & - & - & $0.2 \pm 1.0$ & $58.6 \pm 22.9$ & $2.3 \pm 14.2$ \\
\hline$D_{2}^{+} D^{-}$ & - & - & - & $11.7 \pm 21.1$ \\
\hline
\end{tabular}

\section{Summary}

In this paper we used a coupled-channel approach to perform a simultaneous fit for the data in the major open-charm channels measured by Belle in a wide energy range $\sqrt{s}=3.7 \div 4.7 \mathrm{GeV}$. The main advantage of the approach used here as compared to previous works is that unitarity of the amplitude is under control at every stage of the data analysis. In particular, unitarity is preserved up to the minor contributions of the neglected strange-charm and many-body channels. This allows one to link tightly the parameters in different channels and, as a result, to considerably reduce the number of free parameters of the fit. The main conclusion of the paper is that the suggested method is indeed able to explain all data sets simultaneously and provides a very good overall description of the line shapes. The will definitely improve after consideretion the next update for the data in the open-charm channels [39].

The work is supported by the Russian Foundation for Basic Research (Grant No. 17-0200485).

\section{References}

[1] J. Siegrist et al. et al. (MARK-I Collab.), Phys. Rev. Lett. 36, 700 (1976). 
[2] P. A. Rapidis et al. (MARK-I Collab.), Phys. Rev. Lett. 39, 526 (1977), Erratum-ibid. 39, 974 (1977).

[3] R. Brandelik et al. (DASP Collab.), Phys. Lett. B 76, 361 (1978).

[4] W. Bacino et al. (DELCO Collab.), Phys. Rev. Lett. 40, 671 (1978).

[5] R. H. Schindler et al. (MARK-II Collab.), Phys. Rev. D 21, 2716 (1980).

[6] K. K. Seth, Phys. Rev. D 72, 017501 (2005).

[7] A. Osterheld et al. (Crystal Ball Collab.), SLAC-PUB-4160, (1986).

[8] J. Z. Bai et al. (BES Collab.), Phys. Rev. Lett. 88, 101802 (2002).

[9] M. Ablikim et al. (BES Collab.), Phys. Lett. B 660, 315 (2008).

[10] B. Aubert et al. (BABAR Collab.), Phys. Rev. Lett. 95, 142001 (2005).

[11] B. Aubert et al. (BaBar Collab.), arXiv:0808.1543, (2008).

[12] B. Aubert et al. (BaBar Collab.), Phys. Rev. Lett. 98, 212001 (2007).

[13] G. Pakhlova et al. (Belle Collab.), Phys. Rev. Lett. 101, 172001 (2008).

[14] E. Eichten, K. Gottfried, T. Kinoshita, K. D. Lane, and T. M. Yan, Phys. Rev. D 21, 203 (1980).

[15] G. Pakhlova et al. (Belle Collab.), Phys. Rev. D 77, 011103 (2008).

[16] K. Abe et al. (Belle Collab.), Phys. Rev. Lett. 98, 092001 (2007).

[17] G. Pakhlova et al. (Belle Collab.), Phys. Rev. Lett. 100, 062001 (2008).

[18] G. Pakhlova et al. (Belle Collab.), Phys. Rev. D 80, 091101 (2009).

[19] G. Pakhlova et al. (Belle Collab.), Phys. Rev. D 83, 011101 (2011).

[20] B. Aubert et al. (BABAR Collab.), Phys. Rev. D 76, 111105 (2007).

[21] B. Aubert et al. (BABAR Collab.), Phys. Rev. D 79, 092001 (2009).

[22] P. A. Sanchez et al. (BABAR Collab.), Phys. Rev. D 82, 052004 (2010).

[23] D. Cronin-Hennessy et al. (CLEO Collab.), Phys. Rev. D 80, 072001 (2009).

[24] K. A. Olive et al. (Particle Data Group Collab.), Chin. Phys. C 38, 090001 (2014).

[25] H. B. Li, X. S. Qin and M. Z. Yang, Phys. Rev. D 81, 011501 (2010).

[26] X. Cao and H. Lenske, arXiv:1408.5600 [nucl-th].

[27] X. Cao and H. Lenske, arXiv:1410.1375 [nucl-th].

[28] A. Limphirat, W. Sreethawong, K. Khosonthongkee, and Y. Yan, Phys. Rev. D 89, 054030 (2014).

[29] N. N. Achasov and G. N. Shestakov, Phys. Rev. D 86, 114013 (2012).

[30] N. N. Achasov and G. N. Shestakov, Phys. Rev. D 87, 057502 (2013).

[31] Y. J. Zhang and Q. Zhao, Phys. Rev. D 81, 074016 (2010).

[32] J. Segovia, D. R. Entem, and F. Fernandez, Phys. Rev. D 83, 114018 (2011).

[33] J. Segovia, D. R. Entem, F. Fernandez, and E. Hernandez, Int. J. Mod. Phys. E 22, 1330026 (2013).

[34] E. van Beveren, G. Rupp, and J. Segovia, Phys. Rev. Lett. 105, 102001 (2010).

[35] T. V. Uglov, Y. S. Kalashnikova, A. V. Nefediev, G. V. Pakhlova and P. N. Pakhlov, JETP Lett. 105, 1 (2017).

[36] Durham database, http://durpdg.dur.ac.uk/review/rsig/BELLE.shtml

[37] C. Hanhart, Yu. S. Kalashnikova, P. Matuschek, R. V. Mizuk, A. V. Nefediev, and Q. Wang, Phys. Rev. Lett. 115, 202001 (2015).

[38] F.-K. Guo, C. Hanhart, Yu. S. Kalashnikova, P. Matuschek, R. V. Mizuk, A. V. Nefediev, Q. Wang, and J.-L. Wynen, Phys. Rev. D 93, 074031 (2016).

[39] V. Zhukova et al. (Belle Collab.), Phys. Rev. D 97 1, 012002 (2018). 